\title{
Variational Particle Number Approach for Rational Compound Design
}

\author{
O. Anatole von Lilienfeld, Roberto D. Lins, and Ursula Rothlisberger \\ Laboratoire de Chimie et Biochimie Computationelle, Ecole Polytechnique Fédérale de Lausanne (EPFL), \\ CH-1015 Lausanne, Switzerland
}

(Received 1 April 2005; published 7 October 2005)

\begin{abstract}
Within density functional theory, a variational particle number approach for rational compound design (RCD) is presented. An expression for RCD is obtained in terms of minimization of a suitably defined energy penalty functional whose gradients are the nuclear and the electronic chemical potential. Using combined quantum and molecular mechanics, a nonpeptidic anticancer drug candidate is designed.
\end{abstract}

DOI: 10.1103/PhysRevLett.95.153002

The design of optimized compounds with specific molecular properties is a central goal of many research fields in chemistry, material sciences, and pharmacy. However, even in silico a systematic screening of chemical space for interesting properties is beyond any current capacity [1]. Chemical space is the high-dimensional molecular space spanned by all the possible combinations (stoichiometries) and configurations (isomers) of electrons and nuclei. In general terms, compound design efforts usually attempt a mapping of a given molecular system (defined by its Hamiltonian $\mathcal{H}$ ) to the observable of interest $(\mathcal{O})$. Then, key variables have to be identified which allow one to drastically reduce chemical space to a relevant and small subspace. Once the information is obtained of how $\mathcal{H}$ needs to be modified such that the observable of interest approaches a desired molecular property $\left(\mathcal{O}_{0}\right)$, one attempts to invert the initial mapping,

$$
(\mathcal{H} \mapsto \mathcal{O}) \stackrel{\text { RCD }}{\longleftrightarrow}\left(\mathcal{O}_{0} \mapsto \mathcal{H}_{0}\right) .
$$

Therefore, one tries to identify an optimal system $\mathcal{H}_{\text {opt }}$ simply via $\mathcal{O}_{0} \approx \mathcal{O}_{\text {opt }} \mapsto \mathcal{H}_{\text {opt }}$. Within conventional rational drug design for instance, the interaction of a given drug candidate $(\mathcal{H})$ to a protein is often analyzed in much detail in order to know how to increase its binding affinity $(\mathcal{O})$ via structural modifications. Thus, the underlying interest in a given system $(\mathcal{H})$ is usually due to its interesting properties and their potential tuning by variation of $\mathcal{H}$ in its chemical space.

A large variety of approaches exist that address this inversion problem, ranging from empirical-statistical quantitative structure property relationships to classical force field and semiempirical calculations [2]. The analytical expression for the potential energy in empirical force fields and the fact that the free energy is an equation of state has motivated studies connecting different molecules reversibly through a coupling parameter, commonly called $\lambda$. Thermodynamic integration and other approaches to relative free energies have been successfully carried out ever since Kirkwood's seminal paper in 1935 [3,4]. However, to declare $\lambda$ a dynamical variable in the framework of a statistical mechanics scheme and to use it for compound design has been implemented only a decade ago
PACS numbers: 31.15.Ew, 71.15.Pd, 81.05.Zx, 87.15.Aa

$[5,6]$. Unfortunately, force-field approaches are intrinsically limited due to their classical and empirical nature. Specifically, they will fail to describe all those regions in chemical space where electronic structure effects occur or which have not yet been parametrized. Consequently, they do not allow one to freely explore chemical space for compound design. Using quantum mechanics, it is possible to optimize deterministically $\mathcal{H}$ throughout chemical space and thereby also to carry out de novo compound design in the sense of Refs. [7-9]. However, even a detailed and accurate understanding of the left-hand expression in Eq. (1) does not yet necessarily lead to an inversion of the mapping. Consequently, current applications of first principles methods are quite heuristic.

While a general inversion of the mapping of $\mathcal{H} \mapsto \mathcal{O}$ is not possible (different $\mathcal{H}$ 's can have a comparable property), we attempt here to recursively invert the mapping locally (local in chemical space). That is, starting from a given compound, a nonheuristic formulation for a rational compound design from first principles is presented. The scheme iteratively minimizes a penalty functional in particle space using standard density functional theory (DFT) [10-12] within a grand-canonical ensemble (GCE) notation. For demonstration purposes the presented approach is applied to the design of a nonpeptidic inhibitor of the anticancer target human XIAP (X-chromosome linked inhibitor-of-apoptosis-proteins), which are overexpressed in cancer cells [13-16].

Within DFT and the Born-Oppenheimer approximation to the adiabatic separation of the nuclear and electronic wave function, any ground-state observable $\mathcal{O}$ is a functional of the electron density $n$. The latter is for a fixed number of electrons $N_{e}$ up to a constant in a one-to-one relationship to the external potential $v$ [10]. For an unperturbed system, $v$ is a simple functional of the nuclear charge distribution $Z: v[Z](\mathbf{r})=-\int d \mathbf{r}^{\prime} Z\left(\mathbf{r}^{\prime}\right) /\left|\mathbf{r}-\mathbf{r}^{\prime}\right|$. In the classical point charge limit $Z(\mathbf{r})=Z_{I} \delta\left(\mathbf{r}-\mathbf{R}_{I}\right)$, where $Z_{I}$ is the number of protons at $\mathbf{r}=\mathbf{R}_{I}$, i.e., the atomic number of atom $I$. Consequently, the observable $\mathcal{O}$ can be seen as a function of $N_{e}$ and as a functional of $Z, \mathcal{O}[Z]\left(N_{e}\right)$.

In analogy to the variational optimization of atom centered potentials for molecular properties [17], one can 
define an expression for rational compound design via a penalty functional $\mathcal{P}$, which is to be minimized iteratively by gradient based variations of $N_{e}$ and $Z(\mathbf{r})$,

$$
\min _{N_{e}, Z} \mathcal{P}[Z]\left(N_{e}\right)=\min _{N_{e}, Z}\left|\mathcal{O}[Z]\left(N_{e}\right)-\mathcal{O}_{0}\right|^{2} .
$$

$\mathcal{P}[Z]\left(N_{e}\right)$ penalizes deviations of $\mathcal{O}[Z]\left(N_{e}\right)$ from a desired reference property $\mathcal{O}_{0}$. Equation (2) turns rational compound design into a minimization problem in the discrete chemical space spanned by electrons and nuclei. As in all iterative minimization problems in high-dimensional spaces, analytical gradients offer a fundamental gain in efficiency with respect to screening techniques or finite differences. In this sense $\delta \mathcal{P} / \delta Z$ and $\partial \mathcal{P} / \partial N_{e}$ - available from first principle - can be used within all sorts of gradient based minimization schemes like Newton's rule or conjugate gradient methods [18].

Consider $\mathcal{O}[Z]\left(N_{e}\right)$ to be the GCE energy functional $E[Z]\left(N_{e}\right)$; i.e., our system $\mathcal{H}$ is in contact with an electron and proton particle reservoir, and $\mathcal{O}_{0}=E_{0} \leq E[Z]\left(N_{e}\right)$, then Eq. (2) reduces to the zero temperature GCE variational theorem [19]. The electronic GCE energy $E[Z]\left(N_{e}\right)$ is obtained from the stationary principle for atoms and molecules at a fixed $Z$ [20]: $\delta\left\{E[Z]\left(N_{e}\right)-\mu_{e} N_{e}\right\}=0$, where $E[Z]\left(N_{e}\right)=\int d \mathbf{r} n v+F_{G C}[n] . F_{G C}[n]$ is the universal, only implicitly dependent on $Z$, GCE ground-state functional, while $\mu_{e}$ is a Lagrange multiplier attached to the constraint that $N_{e}=\int d \mathbf{r} n$ and is called the electronic chemical potential $\left[\mu_{e}=\delta E[Z]\left(N_{e}\right) / \delta n(\mathbf{r})=\partial E[Z]\left(N_{e}\right) /\right.$ $\left.\partial N_{e}\right] . \mu_{e}$ is of central importance in DFT and is greatly exploited within conceptual DFT [21]. After minimization of $E[Z]\left(N_{e}\right)$ with respect to $n$, the total potential energy of a system can be given as a functional of $Z, E^{\text {tot }}[Z]\left(N_{e}\right)=$ $E[Z]\left(N_{e}\right)+1 / 2 \int d \mathbf{r} d \mathbf{r}^{\prime} Z(\mathbf{r}) Z\left(\mathbf{r}^{\prime}\right) /\left|\mathbf{r}-\mathbf{r}^{\prime}\right|$.

In analogy to the electronic chemical potential, we introduce here a nuclear chemical potential, $\mu_{n}$, which explicitly measures the tendency of the molecule to vary its nuclear structure $Z$. Unlike $\mu_{e}$ (which corresponds to the molecular electronegativity [22]), $\mu_{n}$ is not a constant for each molecule but a function of space because variations of the ionic charge distribution are allowed everywhere in space,

$$
\begin{aligned}
\mu_{n}(\mathbf{r})= & \frac{\delta E^{\text {tot }}}{\delta Z(\mathbf{r})}=-\int d \mathbf{r}^{\prime}\left(\frac{n\left(\mathbf{r}^{\prime}\right)}{\left|\mathbf{r}-\mathbf{r}^{\prime}\right|}+v\left(\mathbf{r}^{\prime}\right) n^{(1)}\left(\mathbf{r}, \mathbf{r}^{\prime}\right)\right) \\
& +\frac{\delta F_{G C}[n]}{\delta Z(\mathbf{r})}+\int d \mathbf{r}^{\prime} \frac{Z\left(\mathbf{r}^{\prime}\right)}{\left|\mathbf{r}-\mathbf{r}^{\prime}\right|} .
\end{aligned}
$$

Here, $n^{(1)}\left(\mathbf{r}, \mathbf{r}^{\prime}\right)$ denotes the derivative $\delta n\left(\mathbf{r}^{\prime}\right) / \delta Z(\mathbf{r})$, which can be approximated within the linear response in the electronic structure $n\left(\mathbf{r}^{\prime}\right)$ of the system being induced by a local infinitesimally small variation of the ionic charge distribution $Z(\mathbf{r}) \mapsto Z(\mathbf{r})+d Z: n^{(1)}=\sum_{i=1}^{N}\left[\phi_{i}^{*(0)} \phi_{i}^{(1)}+\right.$ $\left.\phi_{i}^{*(1)} \phi_{i}^{(0)}\right]$. This perturbed density is available within CPMD [23] using the density functional perturbation module [24] and the perturbation Hamiltonian of Ref. [17]. $\delta F_{G C}[n] / \delta Z(\mathbf{r})$ consists of the functional derivatives of the
Coulomb energy, of the Kohn-Sham expression for the kinetic energy of the electrons, and of the exchangecorrelation energy with respect to $Z$. However, for a discrete ionic charge distribution and within first order perturbation theory, Eq. (3) reduces simply to the electrostatic field at $\mathbf{r}$,

$$
\mu_{n}(\mathbf{r}) \approx E^{(1)}(\mathbf{r})=-\int d \mathbf{r}^{\prime} \frac{n\left(\mathbf{r}^{\prime}\right)}{\left|\mathbf{r}-\mathbf{r}^{\prime}\right|}+\sum_{I} \frac{Z_{I}}{\left|\mathbf{r}-\mathbf{R}_{I}\right|} .
$$

Within first order perturbation theory the derivative of the energy with respect to changes in atomic number was already presented [25,26]. However, here we consider the explicit molecular response upon an infinitesimal small variation of the nuclear charge distribution everywhere in space, which goes beyond the usual expression for the molecular response upon a variation in the external potential, namely $\delta E / \delta v=n$. While the validity of Kohn-Sham DFT for the unphysical regime of pure electronic states involving noninteger occupation numbers is by now well established [27,28], fractional atomic numbers (classical point charges) are less problematic.

As an illustration, atomic potential energies $E\left[Z_{I}\right]\left(N_{e}\right)$ have been computed using DFT and the BLYP functional [29-32] for fractional number of particles (Fig. 1). The derivative discontinuity at integer values of $N_{e}$, as expected for the exact yet unknown exchange-correlation potential [20], is not reproduced by the employed approximation to the exchange-correlation functional [32]. However, the typical convex shape of the isoprotonic curves is reproduced. The isoelectronic curves are concave, which is in agreement with Ref. [22]. The slope of the $E\left[Z_{I}\right]\left(N_{e}\right)$ surface in Fig. 1 corresponds hence to linear combinations of $\mu_{n}\left(\mathbf{r}=\mathbf{R}_{I}\right)$ and $\mu_{e}$. Note that the system is significantly more sensitive to variations of $Z$ than of $N_{e}$.

Within a GCE formalism $\mu_{n}$ and $\mu_{e}$ represent the respective response of a given system in contact with particle reservoirs upon variation of its electronic or its ionic structure. Higher derivatives of the electronic and nuclear chemical potentials define reactivity response functions and indices in the spirit of conceptual DFT
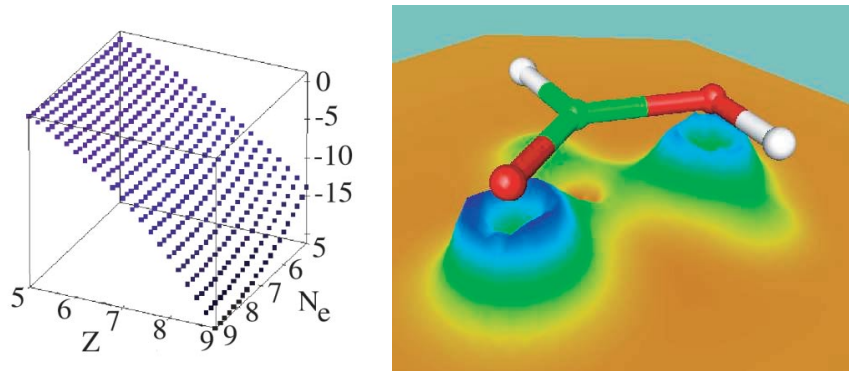

FIG. 1 (color). Left: Atomic potential energy (a.u.) [32] versus the number of electrons $N_{e}$ and the atomic number $Z\left(\mathbf{r}=\mathbf{R}_{I}\right)$. Fractional independent changes of 0.2 for $Z$ and $N_{e}$ range from boron $\left(N_{e}=5, Z=5\right)$ up to fluorine $\left(N_{e}=9, Z=9\right)$. $E\left(N_{e}=\right.$ $\left.5, Z_{I}=5\right)$ (boron) is set to zero, and all other values refer to it. Right: 3D projection of the electrostatic potential $\left[\approx \mu_{n}(\mathbf{r})\right.$, Eq. (4)] in the molecular plane of formic acid. 
[21]. For ground-state systems, further features of $\mu_{n}$ can be established, (i) $\mu_{n}\left(\mathbf{r} \neq \mathbf{R}_{I}\right)$ is related to the proton affinity at $\mathbf{r}$ for the given geometry $\left\{\mathbf{R}_{I}\right\}$. As an illustration, a projection of $\mu_{n}\left(\mathbf{r} \neq \mathbf{R}_{I}\right)$ in the molecular plane of formic acid is presented in Fig. 1. As intuition would suggest, the values are largest close to the oxygen atoms. Note that for pseudopotential or valence electron density calculations the results at $\mathbf{r} \approx \mathbf{R}_{I}$ are nonphysical. (ii) All $\mu_{n}\left(\mathbf{r}=\mathbf{R}_{I}\right)$ are cusps. They measure the transmutational tendency for each atom $I$ in a molecule. We suggest to call $\mu_{n}\left(\mathbf{r}=\mathbf{R}_{I}\right)$ an alchemical potential. (iii) Chemically seen, $-\mu_{n}\left(\mathbf{r}=\mathbf{R}_{I=\mathrm{H}}\right)$ measures a static acidity, i.e., the tendency of the molecule to annihilate the proton. (iv) For pseudopotential DFT calculations, we approximate the alchemical potential in Eq. (3) not by Eq. (4) but by the expectation value of the perturbation Hamiltonian $\mathcal{H}_{\mathrm{I}}^{\prime}$ and the unperturbed electronic structure, $\mu_{n}\left(\mathbf{r}=\mathbf{R}_{I}\right) \approx$ $E_{I}^{(1)}=\left\langle\mathcal{H}_{\mathrm{I}}^{\prime}\right\rangle=\left\langle\sum_{j} \partial_{\sigma_{j}} V_{I}^{P P}\left(\left\{\sigma_{i}\right\}\right)\right\rangle$, where we use the parameter $(\sigma)$ dependent analytical pseudopotentials $V_{I}^{P P}\left(\left\{\sigma_{i}\right\}\right)$ of Goedecker et al. [33]. Here, $\partial_{Z_{I}} V_{I}^{P P}\left(\left\{\sigma_{j}\right\}\right)=$ $-1 /\left|\mathbf{r}-\mathbf{R}_{I}\right| \operatorname{erf}\left[\left|\mathbf{r}-\mathbf{R}_{I}\right| / \sigma_{1}\right]$ needs to be added to the perturbation Hamiltonian given explicitly in Ref. [17]. In this study, $\mu_{n}\left(\mathbf{r}=\mathbf{R}_{I}\right)$ is hence approximated by the sum of the first order perturbations of the energy induced by infinitesimal variations $\left\{\sigma_{j} \mapsto \sigma_{j}+d \sigma_{j}\right\}$ and has been implemented into CPMD [23]. For an atom it was found to grasp roughly $90 \%$ of a finite difference result.

In principle, for the purpose of drug design, all relevant properties that define the druggability of a compound should be included in the penalty formalism of Eq. (2) and $a b$ initio molecular dynamics (MD) should be carried out in order to include all finite temperature effects. However, without any loss of generality, here only the inhibitor's binding affinity is considered as observable, and an experimentally known suitable inhibitor compound is used as the initial structure. Specifically, in the case of XIAP, effective tetrapeptidic inhibitors were identified in vitro through combinatorial screening of the amino acid positions 1, 2, and 4 of the AVPI [Fig. 2(a)] sequence [35]. Although the resulting peptidic inhibitor ARPF [Fig. 2(b)] presents a high affinity $\left(\mathrm{IC}_{50}\right.$ of subnanomolar concentration), such peptidic sequences would be easily cleaved in vivo by proteases, which prohibits any druggability. To our knowledge, no nonpeptidic XIAP inhibitor has been proposed up to date, i.e., a nonpeptidic structure, $\mathcal{H}_{\text {opt }}$, is needed which maximizes as observable, $\mathcal{O}$, the energy of interaction, $E^{\text {int }}=E^{\text {com }}-E^{\text {inh }}-E^{\text {prot }}$. Here, $E^{\mathrm{com}}$ represents the total potential energy of the complex of the inhibitor bound to the protein, and $E^{\text {prot }}$ and $E^{\text {inh }}$ correspond to the energies of the isolated protein and the inhibitor, respectively. We use for the corresponding penalty the gradient with respect to the nuclear charge distribution of the inhibitor $\left(Z^{\text {inh }}\right), \quad \delta \mathcal{P} / \delta Z=\delta E^{\mathrm{com}} /$ $\delta Z^{\text {inh }}-\delta E^{\text {inh }} / \delta Z^{\text {inh }}$.

For the design of a nonpeptidic inhibitor derived from ARPF only independent transmutations of the 9 second

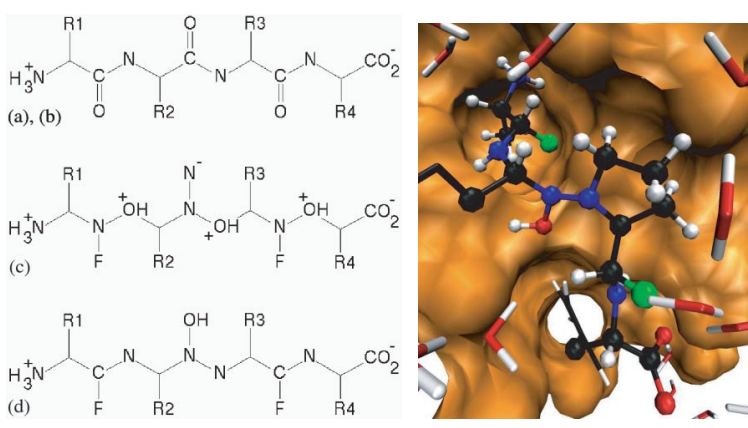

FIG. 2 (color). Left: Topological sketches of inhibitor structures. Upper panel: Peptidic [(a) AVPI: R1 = Ala, R2 = Val, $\mathrm{R} 3=$ Pro, R4 = Ile. (b) ARPF: R1 = Ala, R2 = Arg, R3 = Pro, $\mathrm{R} 4=$ Phe]. Middle panel: First guess nonpeptidic ARPF [(c)]. Lower panel: Final guess nonpeptidic ARPF [(d) npARPF]. Terminal and peptidic atoms are specified, hydrogens are partly omitted. Right: The QM-MM [39] optimized geometry of np-ARPF [(d)] in the XIAP binding pocket. Using the alchemical potential atoms of the three peptide bonds of the ARPF [(b)] have been transmutated to O, C, N, and F (respectively red, black, blue, or green spheres).

row atoms $(I=1, \ldots, 9)$ constituting the three peptide bonds that need to be altered (Fig. 2) have been considered. Thus, the gradient reduces to variations at the positions of these atoms only, $\delta \mathcal{P} / \delta Z=\sum_{I=1}^{9}\left(\partial E^{\text {com }} / \partial Z_{I}-\right.$ $\left.\partial E^{\text {inh }} / \partial Z_{I}\right)=\sum_{I=1}^{9}\left[\mu_{n}^{\operatorname{com}}\left(\mathbf{R}_{I}\right)-\mu_{n}^{\text {inh }}\left(\mathbf{R}_{I}\right)\right]$. Supposing that every atomic number can be changed only by $-1,0$, or 1 , there are $\left(3^{9}-7\right)$ possible nonpeptidic mutants. It is hence intractable to fully screen the relevant chemical space and the penalty gradients offer here a way to guide the exploration at a first principles level of theory.

In order to follow these penalty gradients for a relevant configuration $5 \mathrm{~ns}$ of GROMOS96 43A1 [36] force-field based classical MD simulation of the human XIAP have been conducted in order to equilibrate the enzyme-peptide complexes for AVPI [Fig. 2(a)] and ARPF [Fig. 2(b)] [37]. For the computation of the penalty and its gradients, a snapshot of the equilibrated XIAP-ARPF complex has been chosen for an isolated cluster calculation with DFT [32]. This cluster consists out of the inhibitor and all residues of XIAP within $5 \AA$ distance of the peptidic atoms, being saturated with hydrogen atoms. Starting from the highly potent ARPF [Fig. 2(b)], the 9 peptidic inhibitor atoms have been transmutated according to their alchemical potential. After one iteration a new nonpeptidic model compound [Fig. 2(c)] has been obtained that exhibits an enhancement of the interaction energy by roughly $50 \mathrm{kcal} / \mathrm{mol}$. However, DFT calculations show that one step is insufficient: the structure is unstable as a monomer in gas phase. An iterative procedure following the $\left\{\mu_{n}\left(\mathbf{R}_{I}\right)\right\}$ only for one atom per peptide bond, combined with conservation of charge (through saturation with hydrogen atoms), resulted after four steps into another stable nonpeptidic inhibitor compound, np-ARPF [Fig. 2(d)].

Relative to a QM-MM equilibrated wild-type AVPIXIAP complex $[38,39]$, the ARPF and np-ARPF-XIAP 
(Fig. 2, right) complexes yield within QM-MM, respectively, an increase of the averaged interaction energies by -18.9 and $-18.5 \mathrm{kcal} / \mathrm{mol}$. Since only hydrogen atoms have been used to satisfy the valency of the modified peptide bonds, the entropic contributions to the macroscopic binding affinity for ARPF and np-ARPF are expected to be comparable; i.e., the $\mathrm{IC}_{50}$ of the $\mathrm{np}-\mathrm{ARPF}$ can likewise be expected to be in the subnanomolar range.

In conclusion, we have extended conceptual DFT by the chemical potential for nuclei, $\mu_{n}(\mathbf{r})$. We propose to call $\mu_{n}\left(\mathbf{r}=\mathbf{R}_{I}\right)$ the alchemical potential: it measures the tendency of a system to transmutate a given atom I. QM-MM calculations combined with stability tests have allowed one to successfully apply the presented scheme to the rational compound design of a new nonpeptidic XIAP inhibitor with an expected binding affinity comparable to a highly potent tetrapeptidic inhibitor. Overall, the presented approach is general enough to be extended and applied to other compound design problems exploring chemical space in a less heuristic manner.

O. A.v. L. is thankful to M. Coutinho, G. Fichet, G. von Freymann, P. Geerlings, L. Guidoni, J. Hutter, D. Sebastiani, A.P. Seitsonen, I. Tavernelli, M.E. Tuckerman, and Y. Zhang for discussions. This work has been supported by the Swiss National Science Foundation, Grant No. 510573.

[1] P. Kirkpatrick and C. Ellis, Nature (London) 432, 823 (2004).

[2] D.C. Young, Computational Chemistry (Wiley Interscience, New York, 2001).

[3] J. G. Kirkwood, J. Chem. Phys. 3, 300 (1935).

[4] Encyclopedia of Computational Chemistry, edited by P. R. Schleyer, N. Allinger, T. Clark, J. Gasteiger, P. Kollman, H. S. Schaefer III, and P. Schreiner (John Wiley and Sons, New York, 1998).

[5] B. Tidor, J. Phys. Chem. 97, 1069 (1993).

[6] X. Kong and C. L. Brooks III, J. Chem. Phys. 105, 2414 (1996).

[7] C. Wolverton and A. Zunger, Phys. Rev. Lett. 81, 606 (1998).

[8] A. Franceschetti and A. Zunger, Nature (London) 402, 60 (1999).

[9] G. H. Jóhannesson et al., Phys. Rev. Lett. 88, 255506 (2002).

[10] P. Hohenberg and W. Kohn, Phys. Rev. 136, B864 (1964).

[11] W. Kohn and L. J. Sham, Phys. Rev. 140, A1133 (1965).

[12] W. Kohn, A. D. Becke, and R. G. Parr, J. Phys. Chem. 100, 12974 (1996).

[13] D. R. Green, Cell 102, 1 (2000).

[14] S. W. Fesik, Cell 103, 273 (2000).

[15] Y. Shi, Nat. Struct. Biol. 8, 394 (2001).

[16] S. Srinivasula et al., Nature (London) 410, 112 (2001).

[17] O. A. von Lilienfeld, I. Tavernelli, U. Rothlisberger, and D. Sebastiani, J. Chem. Phys. 122, 14113 (2005).

[18] W. H. Press, S. A. Teukoldky, W. T. Vetterling, and B.P. Flannery, Numerical Recipes (Cambridge University Press, Cambridge, U.K., 1992), 2nd ed.
[19] R. G. Parr and W. Yang, Density Functional Theory of Atoms and Molecules (Oxford Science Publications, 1989).

[20] J. P. Perdew, R. G. Parr, M. Levy, and J. L. Balduz, Phys. Rev. Lett. 49, 1691 (1982).

[21] P. Geerlings, F. D. Proft, and W. Langenaeker, Chem. Rev. 103, 1793 (2003).

[22] R. G. Parr, R. A. Donnelly, M. Levy, and W.E. Palke, J. Chem. Phys. 68, 3801 (1978).

[23] J. Hutter et al., Computer code CPMD, version 3.8, Copyright IBM Corp. and MPI-FKF Stuttgart 19902003, http://www.cpmd.org.

[24] A. Putrino, D. Sebastiani, and M. Parrinello, J. Chem. Phys. 113, 7102 (2000).

[25] E. B. Wilson, Jr., J. Chem. Phys. 36, 2232 (1961).

[26] P. Politzer and R. G. Parr, J. Chem. Phys. 61, 4258 (1974).

[27] M.E. Casida, Phys. Rev. B 59, 4694 (1999).

[28] A. Tachibana and R. G. Parr, Int. J. Quantum Chem. 41, 527 (1992).

[29] A. D. Becke, Phys. Rev. A 38, 3098 (1988).

[30] R. Colle and D. Salvetti, Theor. Chim. Acta 37, 329 (1975).

[31] C. Lee, W. Yang, and R. G. Parr, Phys. Rev. B 37, 785 (1988).

[32] For all DFT calculations the BLYP exchange correlation [29-31], the plane-wave basis code CPMD [23], and pseudopotentials ([33] for cluster, [34] for QM-MM calculations) and converged cutoffs have been used.

[33] S. Goedecker, M. Teter, and J. Hutter, Phys. Rev. B 54, 1703 (1996).

[34] N. Troullier and J. L. Martins, Phys. Rev. B 43, 1993 (1991).

[35] R. A. Kipp et al., Biochemistry 41, 7344 (2002).

[36] W. F. van Gunsteren et al., Biomolecular Simulation: The GROMOS96 Manual and User Guide (vdf Hochschulverlag AG, ETH Zurich, 1996).

[37] Starting from the SMAC-XIAP complex NMR averaged structure (protein data bank code: 1G3F) the AVPI and the ARPF have been modeled in the active site. The system is constituted by the BIR3 domain of human XIAP (94 residues), $\mathrm{Zn}^{2+}$ and either AVPI $\left(+2 \mathrm{Na}^{+}\right)$or ARPF $\left(+1 \mathrm{Na}^{+}\right)$as inhibitors. Periodic boundary conditions, with a supercell of $6.6^{3} \mathrm{~nm}^{3}$ with 9077 or 9073 water molecules, have been used. MD has been conducted with an extended GROMOS96 43A1 force field [36] and SPC water model in the NPT $(T=300 \mathrm{~K}, P=1$ bar $)$ ensemble for $5 \mathrm{~ns}(\Delta t=2 \mathrm{fs})$. Temperature and pressure have been coupled to heat and pressure baths. Long range electrostatics has been accounted for with the particle mesh Ewald method.

[38] A. Laio, J. VandeVondele, and U. Rothlisberger, J. Chem. Phys. 116, 6941 (2002).

[39] Within hybrid quantum [31] and molecular mechanics (QM-MM) [38] Born-Oppenheimer MD at $0 \mathrm{~K}$ the geometry has been optimized for the full ARPF and npARPF-protein complexes. The inhibitor main chain constitutes the QM region, while two inhibitor side chains (Arg and Phe), the protein and the water solvent represent the MM region [37]. Monovalent carbon dummy atoms have been used for capping. 